\title{
Alpha-D-Glucopyranose Monohydrate
}

National Cancer Institute

\section{Source}

National Cancer Institute. Alpha-D-Glucopyranose Monohydrate. NCI Thesaurus. Code C61629.

The monohydrate form of the alpha isoform of D-glucopyranose, a synthetic simple monosaccharide that is used as an energy source. D- glucopyranose is oxidized in various tissues under either aerobic or anaerobic conditions through glycolysis; the oxidation reaction produces carbon dioxide, water, and ATP. 